\title{
Captures
}

Figures, théories et pratiques de l'imaginaire

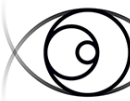

C A P T U R E S

\section{La notion d' «autochtonie»}

\section{dans la littérature et les arts visuels contemporains}

\section{Jean-Philippe Uzel}

Volume 3, Number 1, May 2018

La notion d'«autochtonie»

URI: https://id.erudit.org/iderudit/1055832ar

DOI: https://doi.org/10.7202/1055832ar

See table of contents

Publisher(s)

Figura, Centre de recherche sur le texte et l'imaginaire

ISSN

2371-1930 (digital)

Explore this journal

Cite this document

Uzel, J.-P. (2018). La notion d'«autochtonie» : dans la littérature et les arts visuels contemporains. Captures, 3(1). https://doi.org/10.7202/1055832ar

\section{Article abstract}

This issue sheds light on current debates surrounding the notion of "Indigeneity" in literature and the visual arts, most particularly in Quebec and Canada. At the same time, the articles by Indigenous and Non-Indigenous authors account for the renewed effervescence of contemporary Indigenous creativity. Each of the texts reflect upon what it means to be an Indigenous artist today. They all bring to light the part played by contemporary Indigenous arts in the utterance of Indigenous identity.
This document is protected by copyright law. Use of the services of Erudit (including reproduction) is subject to its terms and conditions, which can be viewed online.

https://apropos.erudit.org/en/users/policy-on-use/ 


\section{La notion d'«autochtonie»}

\section{dans la littérature et les arts visuels contemporains \\ Jean-Philippe Uzel}

Résumé :

Ce numéro, composé de contributions d'auteurs autochtones et allochtones, éclaire les débats actuels entourant la notion d' « autochtonie » dans le champ des arts visuels et de la littérature, tout en rendant compte de la nouvelle effervescence de la création autochtone contemporaine, tout particulièrement au Québec et au Canada. Chacun des textes se penche sur la question de savoir ce que signifie être un artiste autochtone aujourd'hui. Ils mettent tous en évidence que la création contemporaine contribue à l'énonciation de l'identité autochtone.

This issue sheds light on current debates surrounding the notion of "Indigeneity" in literature and the visual arts, most particularly in Quebec and Canada. At the same time, the articles by Indigenous and NonIndigenous authors account for the renewed effervescence of contemporary Indigenous creativity. Each of the texts reflect upon what it means to be an Indigenous artist today. They all bring to light the part played by contemporary Indigenous arts in the utterance of Indigenous identity.

La création autochtone littéraire et visuelle est en ébullition depuis le début des années 2010 . Un des aspects les plus marquants de ce dynamisme artistique est l'affirmation des artistes autochtones sur la scène internationale. L'été 2017 n'a fait que renforcer ce constat puisque les deux plus grands événements internationaux de l'art contemporain, la Documenta de Kassel en Allemagne et la Biennale de Venise, ont accordé une place de premier plan à la création contemporaine autochtone (McMaster: 64). La Documenta est allée même jusqu'à reproduire dans son catalogue une page de la Loi sur les Indiens de 1876, pour mieux en dénoncer le caractère inique (Latimer: 203). L'actualité littéraire n'est pas en reste, tout particulièrement au Québec, où plusieurs écrivains et poètes autochtones sont régulièrement sous les feux de l'actualité?

Cette effervescence s'accompagne toutefois de sa part d'ombre, ou de sa part de vérité diront certains, puisque les cas supposés ou avérés d'appropriation culturelle et de fraude identitaire se multiplient depuis quelque temps 3 . Par exemple, la rétrospective Jimmie Durham. At the Center of the World qui circule à travers les États-Unis et le Canada depuis le début de l'année 2017, a été le théâtre d'une violente polémique sur l'identité cherokee de Jimmie Durham, certainement l'artiste autochtone le plus connu sur la scène internationale de l'art contemporain. Cette controverse (dont plusieurs textes du présent numéro se font l'écho), à laquelle s'ajoutent plusieurs autres 4 , illustre la nécessité de mieux circonscrire la notion d'« autochtonie ». 
Force est de reconnaître que l'« autochtonie ", qui renvoie à la fois à des dimensions culturelles, géopolitiques et historiques, fait l'objet d'importants débats parmi les artistes et les théoriciens autochtones et allochtones. On constate tout d'abord qu'il n'existe aucune définition officielle, seulement des critères communs aux différents peuples autochtones (l'occupation ancestrale du territoire, la marginalisation au sein de la culture majoritaire, la continuité culturelle et linguistique, le sentiment d'appartenance). Les institutions internationales (Nations Unies, Organisation internationale du travail) ne souhaitent pas en proposer une définition trop rigide afin de respecter la diversité des peuples autochtones à l'échelle planétaire. Dans le domaine de l'art et de la littérature, les choses varient grandement d'un pays à un autre. Au Canada et au Québec, les institutions artistiques s'en tiennent à une définition souple de l'autochtonie qui accorde une large place au critère d'autoidentification (voir à ce sujet l'article de Pricile De Lacroix). Aux États-Unis, par contre, l'Indian Arts and Crafts Act exige depuis 1990 de faire la preuve d'une ascendance autochtone reconnue par l'État américain pour pouvoir exposer en tant qu'« Indian Artist » (voir à ce sujet l'article de Jean-Philippe Uzel). Au-delà des questions de lignage, l'usage du terme autochtone continue de susciter des débats dans la sphère artistique et littéraire. Certains chercheurs rejettent purement et simplement cette notion, qui renvoie selon eux à un essentialisme racialiste. D'autres s'interrogent sur la pertinence d'accoler encore le qualificatif « autochtone » à des auteurs et des artistes contemporains dont l'œuvre, reconnue internationalement, n'est en aucune façon réductible à leur identité culturelle. À l'opposé de ces positions critiques et dubitatives, plusieurs jugent que l'essentialisme qui sous-tend la notion est nécessaire pour résister à l'acculturation néocoloniale.

Ce numéro de Captures, composé de contributions d'auteurs autochtones et allochtones, éclaire les débats actuels entourant la notion d'" autochtonie » dans le champ des arts visuels et de la littérature, tout en rendant compte de la nouvelle effervescence de la création autochtone contemporaine, tout particulièrement au Québec et au Canada. Les articles de Guy Sioui Durand et de Marie-Eve Bradette s'appuient tous deux sur des notions autochtones, respectivement le concept wendat d'ohtehrah' (soutien, fondement) et le mot attikamek tikinakan (porte-bébé), pour souligner le dynamisme de la création autochtone, mais également sa résistance face à l'oppression et à la désappropriation du néocolonialisme. Guy Sioui Durand prend l'exemple des œuvres récentes de Caroline Monnet (Algonquine-Française) et de Kent Monkman (Cr-Irlandais) pour montrer qu'il existe chez ces deux artistes une forme de ré-ensauvagement. De son côté, Marie-Eve Bradette se penche sur la poète et slameuse innue Natasha Kanapé Fontaine pour défendre l'idée que l'autochtonie peut se dire et s'écrire en français, autrement dit que l'innu-aimun est une langue en portage s'appuyant et se développant dans la langue française. II est également question d'une jeune poète innue, ou plus exactement « ilnue » (c'est-à-dire une innue de Mashteuiatsh), Marie-Andrée Gill, dans l'article de Luc Vaillancourt et Paul Kawczak. Là encore, il s'agit de savoir comment la langue de l'Autre, celle du colonisateur, peut permettre d'exprimer son identité autochtone. Par une analyse serrée des recueils Béante (2012) et Frayer (2015), les 
auteurs montrent que c'est dans l'affirmation d'une identité plurielle (innue, québécoise, femme, mère) que la poète parvient paradoxalement à exprimer son autochtonie. Le texte de Sophie Guignard traite quant à lui de l'énonciation identitaire par la photographie, plus précisément à travers l'exemple de deux expositions collectives de photographes autochtones, Contemporary Native American Photography(1984) et Steeling the Gaze. Portraits by Aboriginal Artists (2008-2009), présentées respectivement aux États-Unis et au Canada. L'analyse des brochures de ces expositions permet à l'auteure de mettre en évidence la façon dont les photographes autochtones cherchent à s'approprier leur propre représentation et à renverser définitivement la figure de « l'indien imaginaire » forgée par Edward E. Curtis et ses épigones.

Le texte de Pricile De Lacroix se penche pour sa part sur l'importance, au Canada et au Québec, de l'autoidentification comme critère de définition de l'artiste contemporain autochtone. Elle montre, en s'appuyant sur les exemples de deux artistes vivant au Québec, Rita Letendre (Abénakis) et Raymond Dupuis (Malécite), que l'auto-identification offre une grande liberté pour affirmer, ou non, son identité autochtone. Par contraste, elle rappelle que ce critère a peu de poids aux États-Unis, comme l'a illustré la polémique autour des origines cherokees de Jimmie Durham. Dominic Hardy choisit quant à lui une méthode d'enquête particulière : il suit l'odyssée d'une œuvre, Voyage (1988), de l'artiste anishnabé Carl Beam, de sa première apparition en 1989 à l'Art Gallery of Peterborough jusqu'à sa présentation en 2017 dans le cadre du réaménagement de la collection permanente du Musée des Beaux-Arts du Canada. Ce texte, écrit à la première personne, offre une approche très originale des heurs et malheurs de l'institutionnalisation de l'art autochtone au Canada au cours de trois dernières décennies. Le dernier article, celui de Jean-Philippe Uzel, est consacré tout entier à la polémique autour de Jimmie Durham mentionnée plus haut, dont il restitue en détail le contexte. L'auteur essaye de déplacer le débat en montrant que la principale difficulté à laquelle se heurte Jimmie Durham est d'avoir tourné le dos à la Nation Cherokee depuis un quart de siècle, alors que la très grande majorité des artistes autochtones prennent part aujourd'hui au mouvement d'autodétermination et d'affirmation culturelle de leurs communautés respectives.

Les textes de la section « Contrepoints » du numéro offrent un éclairage complémentaire aux articles du dossier en se concentrant, pour la plupart, sur une œuvre d'un artiste des Premières Nations, qui a fait l'objet d'une actualité. Rhonda L. Meier choisit, par exemple, de revenir sur une œuvre emblématique de Jimmie Durham, Self-Portrait (1986), pour repenser la querelle de l'été 2017. Les « contrepoints » de Caroline Nepton Hotte, Geneviève Goyer-Ouimette et Karina Chagnon, sont consacrés à des œuvres d'Eruoma Awashish, Jani Bellefleur-Kaltush, Meky Ottawa, Nadia Myre et Hannah Claus, qui ont toutes fait l'objet d'une exposition à Montréal en 2018. Les contrepoints d'Emily Falvey et Patrice Viau traitent pour leur part d'œuvres qui font déjà partie de l'histoire de l'art contemporain au Canada : celles de Lori Blondeau et Adrian Stimson, et de l'artiste inuit Alootook Ipellie. 
Chacun des textes de ce numéro, articles comme contrepoints, ouvre en fin de compte une perspective singulière sur la notion d'« autochtonie » dans la littérature et les arts visuels contemporains. Ils mettent tous en évidence que la création autochtone contemporaine contribue à l'énonciation de l'identité autochtone. Ce qui retient peut-être le plus l'attention est la capacité de résilience de ces artistes et de ces poètes, lesquels contribuent de façon décisive au dynamisme de la création actuelle, tout en continuant de lutter contre les effets délétères du colonialisme.

1. Nous ne faisons pas ici référence à ce que d'aucuns nomment « la renaissance autochtone » (Bear, 1990), laquelle qualifie le renouveau artistique et spirituel des peuples autochtones des décennies 1970-1980, mais bien à l'effervescence artistique des dernières années, ravivée en 2015 par le dépôt du rapport de la Commission de vérité et réconciliation du Canada, qui accorde une grande place aux créateurs dans le processus de guérison et de réconciliation nationale.

2. Par exemple, le supplément littéraire du journal Le Devoir, Lire, du 21-22 avril 2018, consacrait sa page de couverture à la poète innue Natasha Kanapé Fontaine.

3. Un colloque intitulé «L'appropriation culturelle et les peuples autochtones. Entre protection du patrimoine et liberté de création » a eu lieu à I'UQAM les 4 et 5 avril 2018.

4. On pense par exemple à la polémique qui a eu lieu en décembre 2016 autour des origines autochtones, réelles ou imaginaires, de l'écrivain canadien Joseph Boyden.

\section{Bibliographie}

BEAR, Shirley. 1990. Changers: A Spiritual Renaissance. Wellington : National Indian Arts and Crafts Corporation, $48 \mathrm{p}$.

Latimer, Quinn et Adam SzYmczyk. 2017. The Documenta 14 reader. Munich : Prestel Verlag, 708 p. McMASTER, Gerald. 2017. «Under Indigenous Eyes». Art in America, vol. 105, no 9, p. 64-71. 